\title{
Dendritic cells in lung immunopathology
}

\author{
Peter C. Cook $^{1} \cdot$ Andrew S. MacDonald ${ }^{1}$
}

Received: 6 May 2016 / Accepted: 9 May 2016 / Published online: 2 June 2016

(C) The Author(s) 2016. This article is published with open access at Springerlink.com

\begin{abstract}
Dendritic cells (DCs) lie at the heart of the innate immune system, specialised at recognising danger signals in many forms including foreign material, infection or tissue damage and initiating powerful adaptive immune and inflammatory responses. In barrier sites such as the lung, the instrumental role that DCs play at the interface between the environment and the host places them in a pivotal position in determining the severity of inflammatory disease. The past few years has seen a significant increase in our fundamental understanding of the subsets of DCs involved in pulmonary immunity, as well as the mechanisms by which they are activated and which they may use to coordinate downstream inflammation and pathology. In this review, we will summarise current understanding of the multi-faceted role that DCs play in the induction, maintenance and regulation of lung immunopathology, with an emphasis on allergic pulmonary disease.
\end{abstract}

Keywords Dendritic cells $\cdot$ Lung $\cdot$ Th2 $\cdot$ Th17 $\cdot$ Allergies · Asthma

Dendritic cells (DCs) are critical innate immune cells at barrier sites, including the lung, playing a decisive role in initiation of adaptive immune responses against foreign material, infection, commensals or tissue damage [1]. Of all antigen presenting cell (APC) types in the body, DCs are particularly adept at

This article is a contribution to the special issue on Immunopathology of Lung Diseases - Guest Editors: Tracy Hussell and Aleksander M. Grabiec

Andrew S. MacDonald

andrew.macdonald@manchester.ac.uk

1 Manchester Collaborative Centre for Inflammation Research, Core Technology Facility, The University of Manchester, 46 Grafton Street, M13 9NT Manchester, UK recognising such dangers, migrating from the periphery to the lymphatics, and activating naïve $\mathrm{CD} 4^{+}$and $\mathrm{CD} 8^{+} \mathrm{T}$ cells [2]. While the discussion as to what truly distinguishes DCs from other mononuclear phagocytes continues in some circles, most researchers would agree that functional rather than phenotypic definition of DCs is the key. In this review, in line with the general consensus [1], we define as 'DCs' any mononuclear phagocyte that has the ability to take up antigen (Ag), process it for presentation on MHC-I or II, migrate to the nearest draining lymph node (dLN) and efficiently and effectively activate and polarise naïve $T$ cells. Their crucial role in this regard is clearly illustrated by the fact that people with genetic defects resulting in a lack of DCs, or mice wherein DCs are experimentally depleted, suffer from severely impaired adaptive $\mathrm{T}$ and $\mathrm{B}$ cell responses and increased susceptibility to a wide range of infections [3, 4]. This role as the preeminent cell type in the body for activation and modulation of adaptive responses, and in determining the balance of effector cell vs. regulatory responses, is central to understanding the normal progression of protective immunity in most situations. As clonal expansion of adaptive immunity can directly amplify or regulate immunopathology, consideration of this key role of DCs in maintaining health in the lung is key.

\section{The allergy epidemic and allergic pulmonary disease}

As a mucosal barrier site, the lung is continually exposed to a wide variety of challenges that can trigger immune responses that, when inappropriate or unbalanced, can cause immunopathology. Such responses underpin respiratory disease and are a significant cause of illness and mortality worldwide, with allergic responses leading to asthmatic disease having a dramatic impact on global health [5-7]. Immune mechanisms underlie allergic disease and the pathology associated with 
allergies, typified by a defined range of cell types and pathological responses. These include activation and/or recruitment of a variety of characteristic innate and adaptive immune cells, as well as induction of a range of distinctive physiological processes that together are sometimes termed as 'type 2 inflammation' [8].

In the lung, type 2 inflammatory mechanisms can result in allergic asthma, currently thought to be the predominant cause of asthma in children, and in approximately $50 \%$ of adults [9]. Acute symptoms of asthma include wheezing, coughing and shortness of breath, characterised as airway hyperreactivity (AHR), that can be life threatening. Over time, chronic pulmonary type 2 inflammation can lead to damaging fibrotic pathology and leave individuals at increased risk of secondary pulmonary infections or chronic obstructive pulmonary disease (COPD). Immunological hallmarks of allergic asthma include eosinophilia in patient bronchoalveolar lavage (BAL) fluid, sputum or bronchial biopsies, Th2 polarised $\mathrm{CD}^{+} \mathrm{T}$ cells secreting type 2 cytokines (including IL-4, IL5 and IL-13), and IgE antibody. However, in recent years, as patient stratification approaches have become more refined, it has become clear that asthma is not a single disease and that individuals with asthma can be further classified into distinct groups or 'endotypes' [10]. In particular, not all asthmatics present 'classical' eosinophilic/type 2 disease, with some patients instead displaying disease that is more dominated by neutrophils and insensitive to steroid treatment $[11,12]$. As type 2 (eosinophilic) and type 17 (neutrophilic) inflammation are thought to be governed by different cellular and molecular mechanisms [13], this may help explain the modest levels of success so far of therapies that have tried to target type 2 inflammatory mediators for all asthma patients. Implicit in these processes is the character of the immune and inflammatory response that an individual develops, which directly impacts the type and severity of resulting immunopathology.

Type 2 inflammation is a characteristic of allergic inflammation, but also of parasitic helminth (worm) infection [14-16]. The effector cell types and mechanisms at play in both allergic and helminth infection settings appear to be very similar, being dominated by eosinophilia, $\mathrm{CD} 4^{+} \mathrm{Th} 2$ cells, alternatively activated macrophages and GATA $3^{+}$type 2 innate lymphoid cells (ILC2s). However, it could be argued that, while the type 2 response against parasitic worms has likely evolved 'appropriately' to enable the host and parasite to coexist effectively, the response against allergens is likely an aberrant or 'inappropriate' overexuberant response to foreign material that is not inherently dangerous [17, 18]. Related to this, the type 2 response against worms has also likely evolved to heal tissue damage associated with migration of these often large multicellular organisms through the body, as rapidly and effectively as possible [19-21]. Further, when dysregulated, these very same healing mechanisms can lead to damaging fibrotic pathology [22].
Type 17 inflammation is more characteristic of fungal infection, and typified by neutrophilia and $\mathrm{CD}^{+}{ }^{+} \mathrm{Th} 17$ cells with the ability to make a distinctive range of cytokines, including IL-17A (which we will refer to as IL-17) [23]. Remarkably, although there are numerous triggers of asthma that are abundant in the environment such as pollution, allergens such as house dust mite (HDM), animal dander, pollen and fungal spores, of the 0.5 million deaths attributed to asthma each year worldwide, $50 \%$ may have fungal asthma (GAFFI, 2016). Intriguingly, fungi have the capacity to stimulate both type 2 and type 17 inflammation that may or may not confer immune protection [24-29]. As such, fungal allergens may be of particular interest in trying to understand the mechanisms that dictate the balance of type 2 and type 17 pulmonary disease.

\section{DC activation and induction of adaptive immunity in the lung}

DCs reside throughout the lung tissue, underlying the epithelial layer, poised to encounter foreign material, infections or tissue damage. In this role, they are aided by their ability to actively sample the airways [30]. Although present at low numbers, DCs are highly sensitive to their environment, expressing a variety of pattern recognition receptors (PRRs) including toll-like receptors (TLRs), C-type lectin receptors (CLRs) and Nod-like receptors (NLRs). Through these receptors, they are able to sense a wide range of pathogen, microbe or damage-associated molecules, ranging from bacterial, viral, fungal and protozoal commensals and pathogens through to allergens, particles and pollutants. Additionally, the pulmonary environment presents some unique features relative to other barrier sites, such as specific surfactants and mucins, which can also influence DC activation and function. Importantly, the combination of PRRs engaged can tailor the developing immune response by directly influencing the activation and function of DCs.

\section{The influence of other innate cells on pulmonary DCs}

As well as responding to these environmental stimuli directly through PRR engagement, DCs can also be indirectly activated by other innate cells in the local environment. The pulmonary epithelium is a complex tissue, consisting of a variety of epithelial cell (EC) types including ciliated ECs, goblet cells and Clara cells which vary in composition and distribution depending on their location within the lung [31, 32]. While each of these cell types can act in their own right as innate protection against barrier disruption, infection and damage, their interaction with DCs can also alter the developing adaptive immune response [31]. A range of EC mediators and cytokines, in particular IL-25, IL-33 and thymic stromal 
lymphopoietin (TSLP), is thought to be important for conditioning of DC activation and function and for Th2 induction [33]. In addition, EC-derived endogenous danger-associated molecular patterns (DAMPS) such as ATP [34] and uric acid [35] can also impact DC activation and function in the lung, being triggered in by allergens such as HDM, with the balance of these signals being crucial for mediating allergic inflammation. Though less is known about the role of EC-derived cytokines in the context of neutrophilic asthma, recent evidence confirmed that EC TLR4 expression can mediate eosinophil inflammation in the airways, yet is dispensable for neutrophil influx, which instead depends on haematopoietic TLR4 sources [36]. Furthermore, IL-17 can directly trigger ECs to induce recruitment of neutrophils via CXCL1 [37].

The identity of which specific EC types produce which combinations of mediators in type 2 and type 17 settings, and how this specificity may be selectively controlled, is only beginning to be defined at mucosal sites [38]. Related to this, while EC recognition of endotoxin (bacterial lipopolysaccharide) present in the lung via TLR4 promotes allergic responses [39], chronic exposure to LPS modulates EC responses and dampens DC ability to induce allergic inflammation against HDM [40]. This is a key point, as the lung contains a stable microbiome that is dysregulated during allergic inflammation [41-43]. Further, gnotobiotic mice display exacerbated allergic inflammation [44] and neonatal mice that are yet to establish a diverse microbiota mount enhanced allergic responses to HDM, with the microbiota inducing DC tolerance to allergens [45]. The relevance of this has been demonstrated that exposure to high bacterial environments such as farms in childhood reduces the risk of allergy and asthma [46].

Much less is known about the role of endothelial cells in these processes, though it is clear that angiogenic remodelling is a feature of asthma [47]. It seems likely that they will be heavily involved in settings where danger signals reaching the lung are blood-borne, influencing the migration of immune cells such as eosinophils to and from the lung tissue site [48].

ILCs comprise a diverse grouping of subsets, distinguished based on the transcription factors they express and the cytokines they produce [49]. Although some agreement now exists in terms of ILC genealogy and diversity, vigorous debate still surrounds discussion of their relevance in most settings. Present in very small numbers in the lung, ILC2s expand in response to allergic stimuli and EC production of cytokines such as TSLP and IL-33 [50] and TGF $\beta 1$ [51]. ILC2s can clearly produce large amounts of IL-13 in particular which, in addition to directly impacting pathology, may feed back to influence activation, cytokine/chemokine production and migration of DC subsets from the lung $[52,53]$. In this context, the epidermal growth factor (EGF)-like cytokine amphiregulin (AREG), which can be produced in substantial quantities by ILC2s, has been linked to a variety of outcomes that could directly or indirectly influence immunopathology in the lung [54]. Compared to ILC2s, less is known about how ILC3s may influence development of neutrophilic airway inflammation through production of cytokines such as IL-17. A recent study found that obese mice spontaneously developed AHR with significant numbers of CCR6 $6^{+}$IL- $17^{+}$ILC3s and identified ILC3s in the BAL fluid of asthma patients [55]. It remains to be determined what triggers ILC3 recruitment during asthma, as well as what impact they have on DC ability to promote allergic airway inflammation.

Other innate cell types that can influence developing immunopathology in the lung via their impact on DC activation and function include granulocytes such as eosinophils, basophils and mast cells. For example, eosinophils can impact DC activation and type 2 induction through their degranulation and secretion of granule components such as the alarmin eosinophil-derived neurotoxin or eosinophil peroxidase [56, 57]. Basophils in particular, though dispensable for Th2 induction per se, have been suggested to provide an important accessory cell role as sources of IL-4 in supporting chronic type 2 inflammation [58-61].

\section{DC coordination of innate and adaptive inflammation in the lung}

Depending on the combination of the above factors - PRR engagement, the influence of other innate cells types and the unique lung environment - the outcome will be DC activation or 'maturation' to express a diverse array of surface molecules or mediators that can dramatically influence lung disease development (Fig. 1). The secretion of proinflammatory or antiinflammatory cytokines in response to local activating triggers is the initial, and often overlooked, innate mechanism by which DCs can exert influence over immunopathology in the lung in the very early stages of infection or tissue damage. Although present in very low numbers in the tissues, cytokine production per cell can be substantial [2]. In this way, DCs can rapidly produce high levels of proinflammatory cytokines such as IL-12, TNF $\alpha$ and IL-6 in response to PRR ligands, and in doing so, dramatically shape the character of the local inflammatory environment. For example, DC-derived IL-12 can act directly on NK cells to trigger their activation and IFN $\gamma$ production in what could be deemed a 'bystander' fashion [62].

However, the main mechanism by which DCs impact immunopathology in any setting, including the lung, is through their potent ability to activate and influence adaptive arms of the immune system. Following their activation in the lung, DC migration to the mediastinal LNs enables them to interact with and activate naive $\mathrm{T}$ cells that, through rapid clonal expansion and generation of effector cells that produce high levels of 
cytokine, and immunological memory, can have a major influence over developing and chronic immunopathology.

Effector and memory Th2 cells can exert a range of pathological outcomes in the lung, primarily through the impact of the cytokines they produce. These include granulocyte recruitment, goblet cell hyperplasia (leading to mucus overproduction) and bronchoconstriction [63-65]. Type 2 cytokines, and in particular IL-13, have been proposed to narrow airways in vitro and in vivo by triggering expression of the small GTPase RhoA, which drives muscle contraction [66]. However, other work involving cell-specific deletion of IL-4R $\alpha$ on airway smooth muscle cells has proposed that muscle constriction in type 2 airway inflammation can occur independently of IL-13 [67]. In addition, in asthmatic airways, long-term inflammation results in a dysregulated wound healing response, which causes extensive collagen deposition and subepithelial

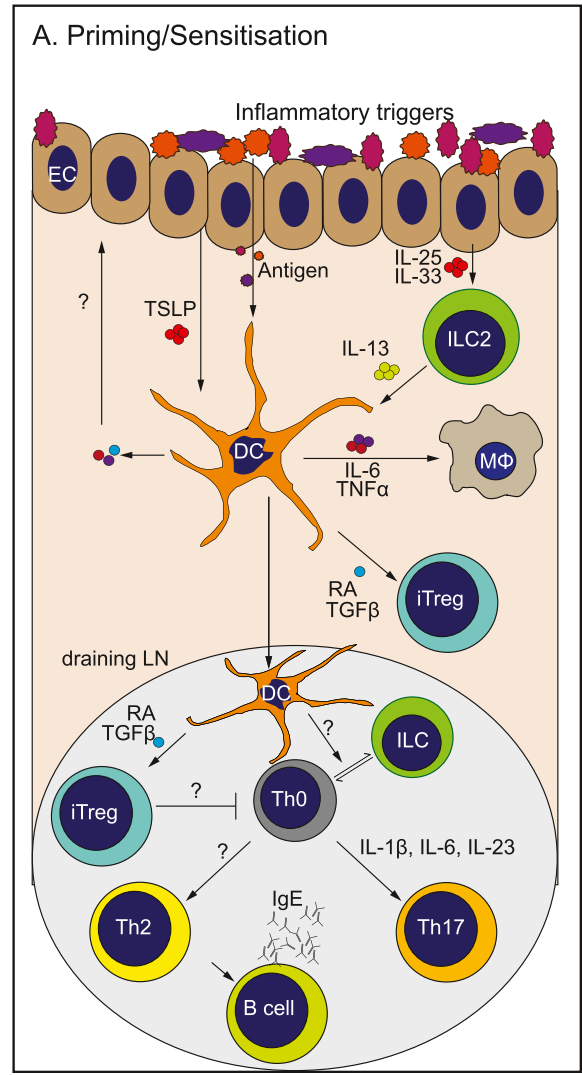

Fig. 1 Key players in type 2/type 17 pulmonary inflammation and immunopathology. a The lung is continually exposed to a variety of signals that can trigger immune responses. In a healthy lung, exposure of epithelial cells (ECs) to danger signals triggers damage responses and the release of tissue factor cytokines such as TSLP IL-25 IL-33 and $\operatorname{TGF} \beta[39,50,51,173]$. Simultaneously, antigens/allergens from the airspaces directly activate dendritic cells (DCs) located in the lung mucosa under the epithelial barrier $[59,86,126]$. The tissue factors released by the epithelium can not only influence the DCs directly but also indirectly via other innate cells such as innate lymphoid cells (ILCs) to alter DC activation or migration and promote allergic inflammation $[52,53]$. Activated DCs can secrete a variety of proinflammatory mediators such as IL- 6 and TNF $\alpha$ that may influence other innate cells in the lung such as intersitial macrophages (Mథs). Simultaneously, these DCs can also secrete regulatory cytokines such as TGF- $\beta$, promoting the generation of inducible regulatory $\mathrm{T}$ cells (iTregs) [152]. DCs then migrate to the draining lymph node (LN) via CCR7 to prime and polarise Th2, Th17 and iTreg cells, a process that may be assisted by cytokine production by accessory cells such as ILCs. The mechanism(s) by which this is achieved, and how these responses are balanced, is not yet clear. b During chronic exposure to inflammatory signals from antigens/allergens and/or innate cells, DCs release chemokines such as CCL17 and CCL22 to recruit effector Th2 and Th17 cells to the lungs,

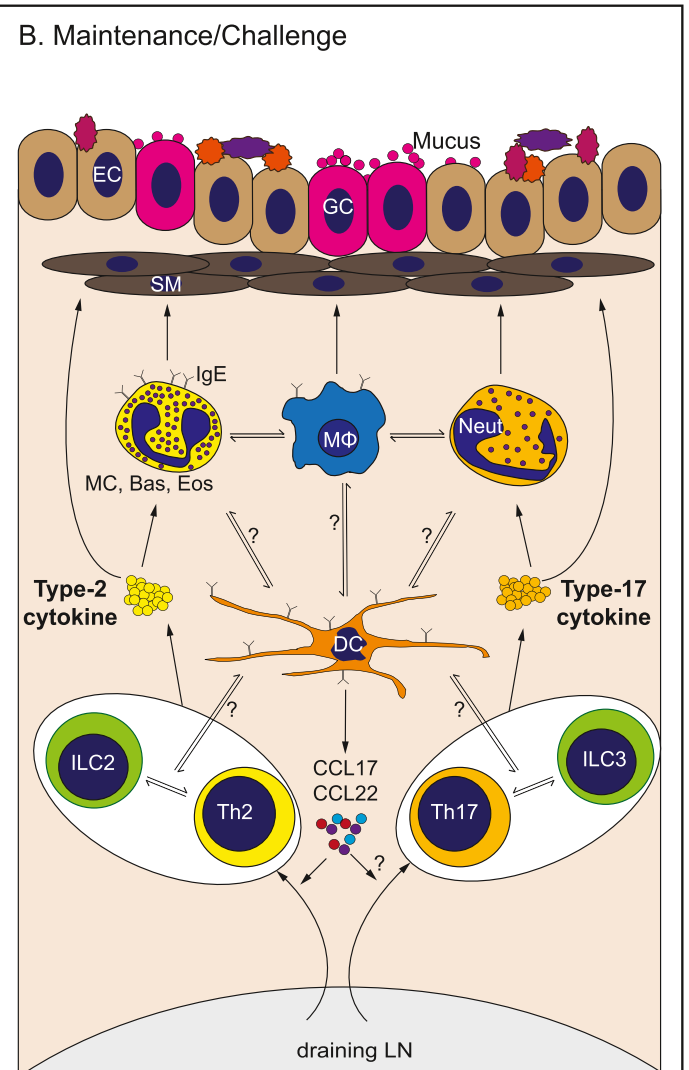

promoting the type $2 /$ type 17 allergic inflammatory environment. It is unknown the extent to which DCs collaborate with other innate cells such as ILCs in the activation of recruited T cell populations, or innate effector cells such as granulocytes (MC; mast cells, Bas; basophils, Eos; eosinophils) and $\mathrm{M} \Phi \mathrm{s}$, to maintain chronic type 2 or type 17 inflammation in the lung. In type 2 settings, this process is also influenced by B cells releasing IgE that can bind to FcR-expressing innate cells. In contrast, type 17 responses promote neutrophil (Neut) infiltration, which may also impact activation and function of innate effector populations such as $M \Phi s$ [174]. In both type 2 and type 17 settings, soluble mediators from innate and adaptive effectors can then mediate disease features such as goblet cell (GC) hyperplasia and smooth muscle (SM) contraction $[63-65,78]$. Thus, DCs play a central role in promoting or regulating chronic pulmonary disease (1) through their central ability to initiate and direct primary responses and (2) through influencing effector cell recruitment and activation during ongoing inflammation. They achieve this through cross-talk with a diverse range of innate and adaptive effector populations, via production of soluble mediators and/or direct cell contact. Defining the specific DC subsets involved and the precise mechanisms they employ to communicate with these diverse effector cells, and how this changes over the course of chronic inflammatory disease and in different disease settings, will reveal new avenues for therapeutic intervention 
fibrosis that involves cytokines such as IL-10 and IL-13 [68]. Further, as well as their direct roles in pathology, some of these Th2-driven outcomes feedback to affect ongoing DC function. For example, the mucin Muc5B has been shown to influence DC maturation in the context of tumour cells [69], though more work is needed to determine how goblet cell hyperplasia and mucins influence DCs to shape allergic inflammation in the lung.

As introduced above, it is increasingly clear that asthma is a broad spectrum of disease and some patients develop a more type 17-associated disease, with dominance of neutrophils rather than eosinophils [70], along with steroid resistance [71]. Several different cellular sources of IL-17 have been implicated in lung inflammation, including Th17 cells, $\gamma \delta \mathrm{T}$ cells, ILCs and even macrophages [72]. The situation is clearly more complex than originally thought, and the mechanisms that determine type 2/type 17 coexistence and balance during lung allergic inflammation are only beginning to be identified. The ability of core elements of type 2 inflammation such as IL-4 [73, 74] or ILC2s [75] to regulate IL-17 is well documented. In contrast, and somewhat counterintuitively, IL-17 may actually promote type 2 responses in some settings [76], including allergic lung inflammation [77]. This suggests a sequential situation where IL-17 may initially promote some aspects of type 2 inflammation, only to be downregulated by type 2 mechanisms once they have established. In addition, IL-17 may be able to directly trigger lung smooth muscle contraction during asthma [78]. More recently, evidence of products of alternatively activated macrophages such as the chitinase-like molecule Ym-1 promoting lung neutrophilia in a type 2 context highlights the complexity of allergic lung pathology [79]. It seems likely that the factors involved in determining the type 2/type 17 balance will be complex and context-dependent. Given that DCs are centrally involved in promotion or regulation of both type 2 and type 17 inflammation, it is likely they will play a decisive role in determining this balance.

\section{How and where do DCs influence pulmonary $T$ cell activation?}

DC migration from the lung to the LNs is a process thought to require upregulation of the chemokine receptor CCR7 and responsiveness to the chemokines CCL19 and 21, which direct DCs to the regions of the LNs where they can interact with and activate naïve T cells $[80,81]$. Once this interaction is initiated, DCs provide naïve T cells with several important activating signals [2]. The first ('signal 1') requires presentation of antigenic peptide to $\mathrm{CD} 4^{+}$or $\mathrm{CD}^{+} \mathrm{T}$ cells in the context of MHC-II or MHC-I, respectively. At this stage, the affinity or avidity of $\mathrm{MHC} / \mathrm{Ag}$ for a particular T cell receptor, and the concentration or dose of Ag, can alter the 'strength' of activating signal delivered to the responding $\mathrm{T}$ cell [82].
Recent development of techniques such as multiphoton microscopy has highlighted the importance of the strength or duration of DC/T cell interaction for naive $\mathrm{T}$ cell activation and polarisation [83]. The second ('signal 2') provides costimulation and survival signals to the responding $\mathrm{T}$ cell, for example through CD80 or CD86 interaction with $\mathrm{T}$ cell CD28. DC provision of signal 1 with inadequate levels of signal 2 can lead to $T$ cell anergy or hyporesponsiveness, rather than activation and clonal expansion, and so is a key step in $\mathrm{T}$ cell activation and generation of functional immunity. In addition to these fundamental $\mathrm{DC} / \mathrm{T}$ cell interactions, further factors at the time of $\mathrm{T}$ cell activation termed 'signal 3' can influence the polarisation of the T cells [84]. Signal 3 generally refers to cytokines produced by DCs that directly influence the naïve $\mathrm{CD} 4^{+} \mathrm{T}$ cell polarisation process, such as IL-12 promotion of Th1 development through T-bet induction and stabilisation. In addition to providing survival and activation signals, some costimulatory molecules may also act to influence $\mathrm{T}$ cell polarisation or function, as has been proposed for CD70 during Th1 induction [85]. Thus, it is the combination of the type and level of expression of signals 1,2 and 3, all 'encoded' in DCs by the nature of stimulus they have encountered in the lung, that is the major determining factor in their $\mathrm{T}$ cell activation and polarising ability in the LNs.

For type 2 immunity, this process likely involves the help of cytokines derived from accessory cells such as ILC2s or basophils, while cells such as ILC3s could conceivably assist DCs in Th17 induction, though this has yet to be shown. In addition, $\mathrm{CD}^{+} \mathrm{T}$ cells activated by DCs provide help via costimulation and cytokine production to B cells to dramatically impact their activation and antibody production. During allergic asthma, type 2 cytokines (particularly IL-4) induce high level IgE production by B cells that can then exert a systemic influence over granulocyte activation in the lung, for example through Fc receptor cross-linking and sensitisation of mast cells leading to heightened release of pathological mediators such as histamine. The cumulative result is clonal expansion of antibody producing $\mathrm{B}$ cells along with effector $\mathrm{T}$ cells that can then leave the LN and migrate back to the lung to exert their influence over the pathological processes at play. Indeed, it has been proposed that pulmonary DC expression of the IgE receptor FceRI is required for their mediation of ongoing HDM responses [86] and promotion of mucous metaplasia [87].

Th2 cell migration from the LNs to the lung tissue is thought to predominantly involve their expression of the chemokine receptor CCR4, increasing responsiveness to the chemokines CCL17 and CCL22 [88, 89]. CCL17 and CCL22 can be produced by a range of innate cells within the lung, including DCs [90, 91], macrophages [92] and ECs [93], are induced by cytokines such as TSLP, IL-25, IL-33 and IL-13 [94-96] and are often found at elevated levels during either murine or human pulmonary allergic inflammation 
[86,91,97-99]. Importantly, although CCR4 is often considered to be particularly important for $\mathrm{Th} 2$ cell recruitment [53, 88], it can be expressed by a variety of other cell types [100], including Th17 cells [101] and ILCs [102], implying that Th17 and ILC recruitment could also involve CCL17 and CCL22. These chemokines may also feedback to influence activation of DCs and ECs, as both have been reported to express CCR4 [103, 104]. Given the variety of cell types that can express CCR4, CCL17 and CCL22, identification of the key producers and responders will be important to better understand migration of effector $\mathrm{CD} 4^{+} \mathrm{T}$ cells to defined regions of the lung tissue during distinct phases of allergic pulmonary inflammation.

While the consensus would be that the majority of adaptive priming by pulmonary DCs occurs in the secondary lymphatic organs [105], tertiary lymphoid structures termed inducible bronchus-associated lymphoid tissue (BALT) can be found in the lung during disease and may provide additional locations for DC/T cell interaction and activation [106]. In fact, it has been suggested that iBALT may even act as a site for naïve $T$ cell priming, in a process that is maintained by and requires DCs $[107,108]$, as well as being a location for enhanced B cell activation. Given increasing awareness of the multifunctionality of other innate cell types such as ECs or ILCs present in the lung tissue, it may be expected that such cells would exert even more of an influence over the process of DC polarisation of naïve T cells in the BBALT than in the more remote LNs, in particular through provision of cytokines that may impact DC functionality, or alter polarisation of responding $\mathrm{T}$ cells directly.

It seems likely that ILCs may play an important accessory role as cytokine sources to enhance immune priming in LNs or iBALT, or influence effector or memory cell function in tissue sites. Further, as ILCs can express MHC [109-111], it remains possible they could act in some settings as APCs, though whether they have the capacity to effectively take up and process Ag, migrate to LNs and express sufficient costimulatory molecules to activate rather than regulate naïve $\mathrm{T}$ cells remains open to debate. Indeed, questions still remain about the relevance of ILCs in general in disease settings: how they are recruited, renewed or the location(s) in vivo where they may interact with and exert their influence over DCs. Determining these factors will be important to enable balanced judgement about their general importance, either directly or indirectly, in the development of immunopathology in the lung, particularly in chronic settings.

\section{DC subsets and induction of type 2 and type 17 inflammation}

Although DCs have been shown to be both sufficient and necessary for Th2 induction in either helminth or allergic type 2 inflammation $[59,112,113]$, the specific DC subset(s) required and the precise mechanisms they employ to promote type 2 inflammation and immunity, remain unclear [14,114-116]. However, some progress has been made on this front in recent years, as the details of DC ontogeny have been unravelled. DCs comprise a heterogeneous variety of cell types or 'subsets', which differ based on tissue location and/or expression of particular surface markers or RNA profiles. These subsets include conventional (or 'classical') DCs (cDCs), plasmacytoid DCs (pDCs) and-particularly during inflammation-non-conventional monocyte-derived DCs (moDCs, also sometimes called TNF $\alpha /$ iNOS producing DCs, or TIP DCs) [117]. In recent years, identification of defining transcription factors has enabled unambiguous classification $\mathrm{cDC}$ and $\mathrm{pDC}$ subsets in both mouse and man [118]. In the mouse, cDCs as defined by expression of the transcription factor Zbtb46 [119, 120] can be separated into two main subsets based on expression of the transcription factors BATF3 or IRF4, whereas pDCs express the transcription factor E2-2 [121]. Batf3 expressing cDCs express $\mathrm{CD} 8 \alpha^{+}$in the spleen and $\mathrm{CD} 103$ or $\mathrm{CD} 24$ in the periphery, whereas $\mathrm{IRF}^{+}{ }^{+} \mathrm{cDCs}$ express $\mathrm{CD} 11 \mathrm{~b}$ and are negative for $\mathrm{CD} 8 \alpha$. In man, $\mathrm{cDC}$ subsets exist expressing CD1c or CD141 in mucosal tissues that appear to be the equivalents of murine $\mathrm{CD} 11 \mathrm{~b}^{+}$and $\mathrm{CD} 8 \alpha^{+} / \mathrm{CD} 103^{+}$subsets respectively [122, 123], with similar expression patterns of transcription factors such as IRF4 in the lung [124].

In the healthy lung, the major populations of DCs to be found are present in the tissue rather than in the airspaces. $\mathrm{CD}_{103^{+}} \mathrm{DCs}$ are heavily associated with the pulmonary epithelium, while the location of $\mathrm{CD}_{11} \mathrm{~b}^{+} \mathrm{DCs}$ is predominantly in the underlying tissue $[125,126]$. Migration studies have shown that, in the murine lung, $\mathrm{CD} 11 \mathrm{~b}^{+}$DCs preferentially acquire and transport soluble $\mathrm{Ag}$, whereas $\mathrm{CD} 103^{+} \mathrm{DCs}$ are more adept at dealing with particulate material [127]. From the limited 'steady state' human lung DC phenotyping data available, equivalent subsets can be identified, again predominantly in the tissues $[122,128]$.

Recently, IRF4-dependent CD11 $\mathrm{b}^{+} \mathrm{cDCs}$ have been associated with both Th17 [124, 129] and Th2 [130, 131] response induction and development. This diversity in $\mathrm{CD} 11 \mathrm{~b}^{+} \mathrm{cDC}$ function likely relates to the heterogeneity that exists within this subset, and the fact that understanding of the transcriptional control of this diversity is still less developed than that of $\mathrm{CD} 8 \alpha^{+} / \mathrm{CD} 103^{+} \mathrm{cDCs}$ or pDCs [132]. Although the precise IRF4-dependent cDC subset responsible for Th2 induction has yet to be unambiguously shown, $\mathrm{CD} 11 \mathrm{~b}^{+} \mathrm{cDCs}$ that are reliant on the transcription factor Klf4, itself downstream of IRF4, have recently been implicated [133]. It is currently unclear how these CD11b ${ }^{+}$IRF4- or Klf4-dependent cDCs relate to the $\mathrm{CD}^{2} 01 \mathrm{~b}^{+} \mathrm{DCs}$ that have also been implicated in Th2 induction against parasitic worm infection and allergic 
responses in the skin [134] and type 17 inflammation to Streptococcus lung infection via production of IL-6 [135].

While it is becoming clear that $\mathrm{CD}_{11} \mathrm{~b}^{+} \mathrm{cDCs}$ may be the dominant $\mathrm{cDC}$ type involved in promotion of type 2 or type 17 inflammation, the role of $\mathrm{CD} 8 \alpha^{+} / \mathrm{CD}_{103}{ }^{+} \mathrm{cDCs}$ and $\mathrm{pDCs}$ in these settings is less well understood. Limited work in this area so far suggests that while $\mathrm{CD} 8 \alpha^{+} / \mathrm{CD} 103^{+} \mathrm{cDCs}$ are particularly adept at promotion of Th1 responses and cross presentation to and activation of $\mathrm{CD} 8^{+} \mathrm{T}$ cells, they are dispensable for Th2 induction [136]. Similarly, while pDCs are characterised by their capacity to produce large amounts of type I IFN in response to viral infection and limited APC ability [137], they do not appear to be vital for Th2 induction against either allergens [138] or helminths [139]. Rather, it appears that both $\mathrm{CD} 8 \alpha^{+} / \mathrm{CD}_{103}{ }^{+} \mathrm{cDCs}$ and $\mathrm{pDCs}$ may in fact help to suppress or counter-regulate type 2 inflammation $[136,138,140]$, though the precise mechanisms involved in this are currently unclear.

\section{DC subsets during inflammation}

In both mouse and human, there is currently a huge disconnect in our understanding of the diversity and impact of DC subsets during any inflammatory setting, including in allergic pulmonary disease: most of our understanding in this area has been developed through study of lung tissue in the steady state, in the absence of overt inflammation. As more refined and higher resolution techniques such as mass cytometry [141], multiparameter flow cytometry and histocytometry [142] are increasingly applied to the lung, our understanding will expand to give vital insight into the diversity, location and activation state of DCs, their interaction with other key cell types and how this may change during disease.

What is likely, in both mouse and human, is that during lung inflammation more DCs can be found in the BAL and the proportion of moDCs present in both BAL and lung tissue increases. In murine models of eosinophilic asthma, CD11 $\mathrm{b}^{+}$ DCs accumulate with effector $T$ cells around the airways following allergen challenge [126]. Additionally, it is clear in such models that $\mathrm{CD} 11 \mathrm{~b}^{+} \mathrm{cDCs}$ are superseded by $\mathrm{CD} 11 \mathrm{~b}^{+}$ moDCs as the predominant DC subset involved in Th2 induction with increasing allergen challenge [86]. Monocytes in general will likely play a much more dominant role in pulmonary inflammation and pathology than is currently appreciated, given increasing awareness of the impact of local and systemic inflammation on conditioning of monocytes early in their bone marrow development that can influence their resulting function in the periphery $[143,144]$ and the multifunctionality of monocytes themselves [145].

As DCs play such a pivotal role in dictating the balance of adaptive immunity, determining in any given disease which subsets predominate, and the profile of activation that they present, may provide vital clues as to the main immunopathologic mechanisms involved. Importantly for asthma and pulmonary

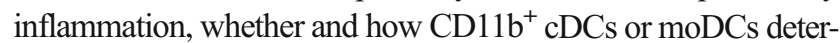
mine the balance between eosinophilic/type 2 and neutrophilic/ type 17 inflammation remains to be determined and may be of particular relevance in situations such as fungal asthma.

Although the importance of DCs in priming and polarisation of naïve T cells in the lung draining LNs is clear, the role of DCs in recruitment, retention or reactivation of pulmonary effector or memory $\mathrm{T}$ cells during chronic inflammation is much less clear. In fact, the fundamentals of Th2 and Th17 cells are currently poorly understood in comparison to Th1 cells or $\mathrm{CD} 8^{+} \mathrm{T}$ cells, with little evidence that current concepts of $\mathrm{T}$ cell expansion, contraction and memory development/longevity are relevant in the context of Th2 or Th17 cells. However, recent work using HDM-specific MHCII tetramers has suggested that a single exposure to HDM generates a central memory population of $\mathrm{CD}^{+} \mathrm{T}$ cells that remain in the lung to mediate AHR [146]. Further, Th2 effector cells have been shown to respond to IL-33 by exacerbating eosinophilic inflammation without the need for APC interaction [147]. Given that pulmonary disease is often chronic in nature, additional factors to consider include the development of T cell hyperresponsiveness over time and whether DCs may play a decisive role in fine tuning of memory $\mathrm{T}$ cell function.

\section{Immunogenic vs. tolerogenic roles for DCs in lung inflammation}

As well as their potent immunogenic ability, DCs can also play a central role in regulation of inflammation and immunopathology [148]. Such 'tolerogenic' DCs are thought to be able to employ a variety of direct and indirect regulatory mechanisms, including secretion of antiinflammatory cytokines, induction of anergy or hyporesponsiveness and promotion of peripheral or induced regulatory T cells (iTregs) [149, 150]. In the steady state, lung DCs appear less able to induce Tregs than macrophages [151]. However, after allergen challenge, $\mathrm{CD}_{103^{+}} \mathrm{DCs}$ become strongly tolerogenic, producing both retinoic acid (RA) and TGF $\beta$ to generate iTregs [152]. In patients with COPD, tolerogenic DCs that induce IL- $10^{+}$ iTregs have been identified [153], and Helicobacter pylori modulation of allergic inflammation is dependent on DC production of IL-10, yet independent of Tregs [154]. Given the ability of DCs to coordinate T cell effector function, including induction of iTregs, this is likely to have a profound impact on immunopathology in the lung involving any kind of adaptive component.

Interesting parallels exist between the phenotype of Th2 promoting DCs and some tolerogenic DCs, in particular a general low level or 'immature' phenotype evident in either case $[114,115]$. Indeed, in some settings, Th2-inducing DCs, through their promotion of type 2 responses that can counter- 
regulate Th1 inflammation, could be considered regulatory or tolerogenic. As yet, a side by side comparison of Th2 vs. tolerogenic DCs has yet to be carried out but would likely be of real value in distinguishing mechanisms that are truly regulatory from those that are Th2 promoting. Further, the definition of immunogenic vs. tolerogenic features of DCs may relate directly to developing concepts of 'innate memory', where 'trained' DCs could display enhanced proinflammatory capacity, whereas 'tolerised' DCs would instead be regulatory, with the outcome being reduced or increased susceptibility to some infections [155], along with more or less severe inflammatory pathology [156]. As in other tissue sites, the nature of the stimuli that DCs encounter in the lung likely determines training vs. tolerance, and so immunogenic vs. tolerogenic capacity.

\section{DC mechanisms of $\mathrm{T}$ cell polarisation}

Despite advances in subset identification and the general association of $\mathrm{CD} 8 \alpha^{+} / \mathrm{CD} 103^{+}$DCs with Th1 [157] and

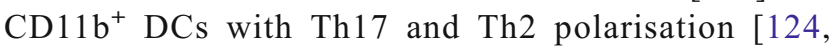
129-131,133,158], the specific mechanisms employed by DCs to promote type 2 inflammation and immunity remain unclear and a key unanswered question in the field $[14,114,115]$. The paradigm established over the past decades is that DC production of IL-12 is central to their ability to promote Th1 polarisation [159], though there are some exceptions to this general rule, such as the costimulatory molecule CD70 compensating for Th1 induction in the absence of IL-12 in some settings [85]. In type 17 responses, DC production of IL- $1 \alpha$, IL- 6 and IL-23 has been strongly linked to their Th17 inductive ability, with variable reliance on each of these mediators depending on context, and when comparing murine and human DCs [160-162]. In terms of DC induction of iTregs, one cytokine that has been particularly implicated is TGF $\beta$, and subsets of DCs can express TGF $\beta$ activating integrin $\alpha \mathrm{V} \beta 8$ to facilitate this process [163]. In this context, as TGF $\beta$ can also act in concert with cytokines such as IL- 6 to promote Th17 induction $[160,161]$, the relative balance of each cytokine at the time of $\mathrm{CD}^{+} \mathrm{T}$ cell priming could result in either a regulatory (iTreg) or an inflammatory (type 17/neutrophilic) outcome. In murine models of HDM or OVAinduced airway inflammation, mice with CD11crestricted deficiency in $\alpha \mathrm{v} \beta 8$ displayed reduced $\mathrm{CD}^{+}$ $\mathrm{T}$ cell IL-17 production but intact $\gamma \delta \mathrm{T}$ cell IL-17, iTreg and Th2 responses [78]. Together, this suggests that the dominant role for DC expression of $\alpha \mathrm{V} \beta 8$ in pulmonary inflammation is promotion of Th17 cells, though its potential role in Th2 induction, and which specific pulmonary DC subset(s) express this TGF $\beta$ activating integrin, currently remains unclear.
In contrast to Th1, Th17 and iTregs, no DC-derived cytokine has yet been convincingly identified that is central to their Th2 induction ability. Indeed, data so far indicate that DCs tend to only display a limited maturation phenotype in response to allergens or helminths, with low level and non-distinctive cytokine production or changes in mRNA profiles, making it difficult to identify candidate mechanisms for type 2 induction $[14,114,115]$. While DC production of IL-4 or IL-10 is not a fundamental requirement for Th2 induction [164-166], it has recently been suggested that DC expression of PDL2 or IL-33, under the control of the transcription factor IRF4, may be important for this process [130, 131].

Although a clear DC-derived signal 3 for Th2 induction remains elusive, it is becoming increasingly clear that epigenetic mechanisms of transcriptional regulation may also play a major role in control of DC activation and function during $\mathrm{T}$ cell activation and polarisation and in allergic airway inflammation. The deubiquitinase A20 has been shown to limit DC activation and restrain their induction of inflammatory responses [167], while the methyl-CpG-binding protein Mbd2 is required for DC promotion of type 2 responses against either helminths or allergens [168]. This epigenetic layer of control of immune cells is not restricted to DCs, with A20, Mbd2 or the demethylase Jmjd3 also playing a part in control of innate cells such as macrophages and mast cells [169, 170], as well as, in some cases, adaptive $\mathrm{T}$ and $\mathrm{B}$ cells $[171,172]$. Thus, defining the processes under the control of epigenetic regulators such as A20 and Mbd2 is an exciting new frontier that is already leading to discovery of previously unappreciated core mechanisms that innate cells such as DCs used in promotion or regulation of pulmonary inflammation.

\section{Conclusions}

Although lung type 2 inflammatory disease can be triggered by a wide range of stimuli, DCs are centrally involved in the initiation and direction of the adaptive immune response and immunopathology that ultimately determines the severity of chronic disease. A better understanding of the DC subsets involved, the mechanisms that they employ and the cell types that they interact with during promotion, maintenance or regulation of inflammation in the lung will lead to the future development of novel therapeutics to combat the damaging immunopathology associated with chronic pulmonary inflammatory disease (Fig. 1). 


\section{Compliance with ethical standards}

Disclosure The MCCIR is a joint venture between the University of Manchester, AstraZeneka and GSK.

Open Access This article is distributed under the terms of the Creative Commons Attribution 4.0 International License (http:// creativecommons.org/licenses/by/4.0/), which permits unrestricted use, distribution, and reproduction in any medium, provided you give appropriate credit to the original author(s) and the source, provide a link to the Creative Commons license, and indicate if changes were made.

\section{References}

1. Merad M, Sathe P, Helft J, Miller J, Mortha A (2013) The dendritic cell lineage: ontogeny and function of dendritic cells and their subsets in the steady state and the inflamed setting. Annu Rev Immunol 31:563-604

2. Banchereau J et al (2000) Immunobiology of dendritic cells. Annu Rev Immunol 18:767-811

3. Collin M, Bigley V, Haniffa M, Hambleton S (2011) Human dendritic cell deficiency: the missing ID? Nat Rev Immunol 11:575583

4. Bar-On L, Jung S (2010) Defining dendritic cells by conditional and constitutive cell ablation. Immunol Rev 234:76-89

5. Masoli M, Fabian D, Holt S, Beasley R (2004) Global Initiative for Asthma (GINA) Program. The global burden of asthma: executive summary of the GINA Dissemination Committee report. Allergy 59:469-478

6. Stock S et al (2005) Asthma: prevalence and cost of illness. Eur Respir J 25:47-53

7. Croisant S (2014) Epidemiology of asthma: prevalence and burden of disease. Adv Exp Med Biol 795:17-29

8. Fahy JV (2014) Type2 inflammation in asthma - present in most, absent in many. Nat Rev Immunol 15:57-65

9. Lambrecht BN, Hammad H (2014) The immunology of asthma. Nat Immunol 16:45-56

10. Choy DF et al (2015) TH2 and TH17 inflammatory pathways are reciprocally regulated in asthma. Sci Transl Med 7:301ra129

11. Green RH et al (2002) Analysis of induced sputum in adults with asthma: identification of subgroup with isolated sputum neutrophilia and poor response to inhaled corticosteroids. Thorax $57: 875-879$

12. McGrath KW et al (2012) A large subgroup of mild-to-moderate asthma is persistently noneosinophilic. Am J Respir Crit Care Med 185:612-619

13. Newcomb DC, Peebles RS (2013) Th17-mediated inflammation in asthma. Curr Opin Immunol 25:755-760

14. Pulendran B, Artis D (2012) New paradigms in type 2 immunity. Science 337:431-435

15. Allen JE, Maizels RM (2011) Diversity and dialogue in immunity to helminths. Nat Rev Immunol 11:375-388

16. Locksley RM (2010) Asthma and allergic inflammation. Cell 140: 777-783

17. Yazdanbakhsh M, Kremsner PG, van Ree R (2002) Allergy, parasites, and the hygiene hypothesis. Science 296:490-494

18. Maizels RM et al (2004) Helminth parasites - masters of regulation. Immunol Rev 201:89-116

19. Gause WC, Wynn TA, Allen JE (2013) Type 2 immunity and wound healing: evolutionary refinement of adaptive immunity by helminths. Nat Rev Immunol 13:607-614
20. Allen JE, Sutherland TE (2014) Host protective roles of type 2 immunity: parasite killing and tissue repair, flip sides of the same coin. Semin Immunol. doi:10.1016/j.smim.2014.06.003

21. Allen JE, Wynn TA (2011) Evolution of Th2 immunity: a rapid repair response to tissue destructive pathogens. PLoS Pathog 7, e1002003

22. Wynn $\mathrm{T}$ (2004) Fibrotic disease and the $\mathrm{T}(\mathrm{H}) 1 / \mathrm{T}(\mathrm{H}) 2$ paradigm. Nat Rev Immunol 4:583-594

23. Brown GD et al (2012) Hidden killers: human fungal infections. Sci Transl Med 4:165rv13

24. Werner JL et al (2009) Requisite role for the dectin-1 beta-glucan receptor in pulmonary defense against Aspergillus fumigatus. $\mathrm{J}$ Immunol 182:4938-4946

25. Zelante $\mathrm{T}$ et al (2007) IL-23 and the Th17 pathway promote inflammation and impair antifungal immune resistance. Eur J Immunol 37:2695-2706

26. Lilly LM et al (2012) The $\beta$-glucan receptor dectin-1 promotes lung immunopathology during fungal allergy via IL-22. J Immunol 189:3653-3660

27. Urb M et al (2015) Evolution of the immune response to chronic airway colonization with Aspergillus fumigatus Hyphae. Infect Immun 83:3590-3600

28. Rivera A et al (2011) Dectin-1 diversifies Aspergillus fumigatusspecific T cell responses by inhibiting T helper type 1 CD4 T cell differentiation. J Exp Med 208:369-381

29. Porter PC et al (2011) Necessary and sufficient role for $\mathrm{T}$ helper cells to prevent fungal dissemination in allergic lung disease. Infect Immun 79:4459-4471

30. Veres TZ et al (2011) Spatiotemporal and functional behavior of airway dendritic cells visualized by two-photon microscopy. Am J Pathol 179:603-609

31. Hammad H, Lambrecht BN (2015) Barrier epithelial cells and the control of type 2 immunity. Immunity 43:29-40

32. Whitsett JA, Alenghat T (2014) Respiratory epithelial cells orchestrate pulmonary innate immunity. Nat Immunol 16:27-35

33. Lambrecht BN, Hammad H (2014) Allergens and the airway epithelium response: gateway to allergic sensitization. J Allergy Clin Immunol 134:499-507

34. Idzko $\mathrm{M}$ et al (2007) Extracellular ATP triggers and maintains asthmatic airway inflammation by activating dendritic cells. Nat Med 13:913-919

35. Kool M et al (2011) An unexpected role for uric acid as an inducer of T helper 2 cell immunity to inhaled antigens and inflammatory mediator of allergic asthma. Immunity 34:527-540

36. McAlees JW et al (2015) Distinct Tlr4-expressing cell compartments control neutrophilic and eosinophilic airway inflammation. Mucosal Immunol 8:863-873

37. Liang SC et al (2007) An IL-17F/A heterodimer protein is produced by mouse Th17 cells and induces airway neutrophil recruitment. J Immunol 179:7791-7799

38. von Moltke J, Ji M, Liang H-E, Locksley RM (2016) Tuft-cellderived IL-25 regulates an intestinal ILC2-epithelial response circuit. Nature 529:221-225

39. Hammad $\mathrm{H}$ et al (2009) House dust mite allergen induces asthma via Toll-like receptor 4 triggering of airway structural cells. Nat Med 15:410-416

40. Schuijs MJ et al (2015) Farm dust and endotoxin protect against allergy through A20 induction in lung epithelial cells. Science 349:1106-1110

41. Charlson ES et al (2012) Assessing bacterial populations in the lung by replicate analysis of samples from the upper and lower respiratory tracts. PLoS ONE 7, e42786

42. Hilty $\mathrm{M}$ et al (2010) Disordered microbial communities in asthmatic airways. PLoS ONE 5, e8578 
43. Huang YJ et al (2011) Airway microbiota and bronchial hyperresponsiveness in patients with suboptimally controlled asthma. J Allergy Clin Immunol 127:372-381.e1-3

44. Herbst $\mathrm{T}$ et al (2011) Dysregulation of allergic airway inflammation in the absence of microbial colonization. Am J Respir Crit Care Med 184:198-205

45. Gollwitzer ES et al (2014) Lung microbiota promotes tolerance to allergens in neonates via PD-L1. Nat Med 20:642-647

46. von Mutius E, Vercelli D (2010) Farm living: effects on childhood asthma and allergy. Nat Rev Immunol 10:861-868

47. Pascual RM, Peters SP (2005) Airway remodeling contributes to the progressive loss of lung function in asthma: an overview. J Allergy Clin Immunol 116:477-86, quiz 487

48. Asosingh $\mathrm{K}$ et al (2016) Eotaxin-rich proangiogenic hematopoietic progenitor cells and CCR $3+$ endothelium in the atopic asthmatic response. J Immunol 196:2377-2387

49. Artis D, Spits H (2015) The biology of innate lymphoid cells. Nature 517:293-301

50. Mohapatra A et al (2015) Group 2 innate lymphoid cells utilize the IRF4-IL-9 module to coordinate epithelial cell maintenance of lung homeostasis. Mucosal Immunol. doi:10.1038/mi.2015.59

51. Denney L et al (2015) Pulmonary epithelial cell-derived cytokine TGF- $\beta 1$ is a critical cofactor for enhanced innate lymphoid cell function. Immunity 43:945-958

52. Halim TYF et al (2014) Group 2 innate lymphoid cells are critical for the initiation of adaptive $T$ helper 2 cell-mediated allergic lung inflammation. Immunity 40:425-435

53. Halim TYF et al (2015) Group 2 innate lymphoid cells license dendritic cells to potentiate memory $\mathrm{TH} 2$ cell responses. Nat Immunol. doi:10.1038/ni.3294

54. Zaiss DMW, Gause WC, Osborne LC, Artis D (2015) Emerging functions of amphiregulin in orchestrating immunity, inflammation, and tissue repair. Immunity 42:216-226

55. Kim HY et al (2014) Interleukin-17-producing innate lymphoid cells and the NLRP3 inflammasome facilitate obesity-associated airway hyperreactivity. Nat Med 20:54-61

56. Yang D et al (2008) Eosinophil-derived neurotoxin acts as an alarmin to activate the TLR2-MyD88 signal pathway in dendritic cells and enhances Th2 immune responses. J Exp Med 205:79-90

57. Chu VT et al (2014) Eosinophils promote generation and maintenance of immunoglobulin-a-expressing plasma cells and contribute to gut immune homeostasis. Immunity 40:582-593

58. Tang $\mathrm{H}$ et al (2010) The $\mathrm{T}$ helper type 2 response to cysteine proteases requires dendritic cell-basophil cooperation via ROSmediated signaling. Nat Immunol 11:608-617

59. Hammad H et al (2010) Inflammatory dendritic cells - not basophils - are necessary and sufficient for induction of Th2 immunity to inhaled house dust mite allergen. J Exp Med 207:2097-2111

60. Ohnmacht $\mathrm{C}$ et al (2010) Basophils orchestrate chronic allergic dermatitis and protective immunity against helminths. Immunity 33:364-374

61. Sullivan BM et al (2011) Genetic analysis of basophil function in vivo. Nat Immunol 12:527-535

62. Reis e Sousa C et al (1997) In vivo microbial stimulation induces rapid CD40 ligand-independent production of interleukin 12 by dendritic cells and their redistribution to T cell areas. J Exp Med 186:1819-1829

63. Wills-Karp M et al (1998) Interleukin-13: central mediator of allergic asthma. Science 282:2258-2261

64. Grunig G et al (1998) Requirement for IL-13 independently of IL4 in experimental asthma. Science 282:2261-2263

65. Kuperman DA et al (2002) Direct effects of interleukin-13 on epithelial cells cause airway hyperreactivity and mucus overproduction in asthma. Nat Med 8:885-889
66. Chiba Y et al (2009) Interleukin-13 augments bronchial smooth muscle contractility with an up-regulation of RhoA protein. Am J Respir Cell Mol Biol 40:159-167

67. Kirstein F et al (2010) Expression of IL-4 receptor alpha on smooth muscle cells is not necessary for development of experimental allergic asthma. J Allergy Clin Immunol 126:347-354

68. Wilson MS et al (2007) IL-13Ralpha2 and IL-10 coordinately suppress airway inflammation, airway-hyperreactivity, and fibrosis in mice. J Clin Investig 117:2941-2951

69. García EP et al (2016) MUC5B silencing reduces chemoresistance of MCF-7 breast tumor cells and impairs maturation of dendritic cells. Int J Oncol 48:2113-2123

70. Manni ML et al (2014) The complex relationship between inflammation and lung function in severe asthma. Mucosal Immunol 7: 1186-1198

71. Woodruff PG et al (2009) T-helper type 2-driven inflammation defines major subphenotypes of asthma. Am J Respir Crit Care Med 180:388-395

72. Song C et al (2008) IL-17-producing alveolar macrophages mediate allergic lung inflammation related to asthma. J Immunol 181: 6117-6124

73. Harrington LE et al (2005) Interleukin 17-producing CD4+ effector T cells develop via a lineage distinct from the T helper type 1 and 2 lineages. Nat Immunol 6:1123-1132

74. Park $\mathrm{H}$ et al (2005) A distinct lineage of CD4 T cells regulates tissue inflammation by producing interleukin 17. Nat Immunol 6: 1133-1141

75. Van Dyken SJ et al (2014) Chitin activates parallel immune modules that direct distinct inflammatory responses via innate lymphoid type 2 and $\gamma \delta$ T cells. Immunity. doi:10.1016/j.immuni. 2014.02.003

76. Allen JE, Sutherland TE, Ruckerl D (2015) IL-17 and neutrophils: unexpected players in the type 2 immune response. Curr Opin Immunol 34:99-106

77. Nakae S et al (2002) Antigen-specific T cell sensitization is impaired in IL-17-deficient mice, causing suppression of allergic cellular and humoral responses. Immunity 17:375-387

78. Kudo $\mathrm{M}$ et al (2012) IL-17A produced by $\alpha \beta$ T cells drives airway hyper-responsiveness in mice and enhances mouse and human airway smooth muscle contraction. Nat Med 18:547-554

79. Sutherland TE et al (2014) Chitinase-like proteins promote IL-17mediated neutrophilia in a tradeoff between nematode killing and host damage. Nat Immunol. doi:10.1038/ni.3023

80. Braun A et al (2011) Afferent lymph-derived T cells and DCs use different chemokine receptor CCR7-dependent routes for entry into the lymph node and intranodal migration. Nat Immunol 12: 879-887

81. Alvarez D, Vollmann EH, von Andrian UH (2008) Mechanisms and consequences of dendritic cell migration. Immunity 29:325342

82. Yamane H, Paul WE (2013) Early signaling events that underlie fate decisions of naive CD4(+) T cells toward distinct T-helper cell subsets. Immunol Rev 252:12-23

83. van Panhuys N, Klauschen F, Germain RN (2014) T-cell-receptordependent signal intensity dominantly controls CD4(+) T cell polarization in vivo. Immunity. doi:10.1016/j.immuni.2014.06.003

84. Kapsenberg ML (2003) Dendritic-cell control of pathogen-driven T-cell polarization. Nat Rev Immunol 3:984-993

85. Soares $\mathrm{H}$ et al (2007) A subset of dendritic cells induces CD4+ T cells to produce IFN- by an IL-12-independent but CD70dependent mechanism in vivo. J Exp Med 204:1095-1106

86. Plantinga $\mathrm{M}$ et al (2013) Conventional and monocyte-derived $\mathrm{CD} 11 \mathrm{~b}(+)$ dendritic cells initiate and maintain $\mathrm{T}$ helper 2 cellmediated immunity to house dust mite allergen. Immunity 38 : $322-335$ 
87. Grayson MH et al (2007) Induction of high-affinity IgE receptor on lung dendritic cells during viral infection leads to mucous cell metaplasia. J Exp Med 204:2759-2769

88. Perros F, Hoogsteden HC, Coyle AJ, Lambrecht BN, Hammad H (2009) Blockade of CCR4 in a humanized model of asthma reveals a critical role for DC-derived CCL17 and CCL22 in attracting Th2 cells and inducing airway inflammation. Allergy 64:995-1002

89. Lloyd CM et al (2000) CC chemokine receptor (CCR)3/eotaxin is followed by CCR4/monocyte-derived chemokine in mediating pulmonary $\mathrm{T}$ helper lymphocyte type 2 recruitment after serial antigen challenge in vivo. J Exp Med 191:265-274

90. Alferink J et al (2003) Compartmentalized production of CCL17 in vivo: strong inducibility in peripheral dendritic cells contrasts selective absence from the spleen. J Exp Med 197:585-599

91. Medoff BD et al (2009) CD11b + myeloid cells are the key mediators of Th 2 cell homing into the airway in allergic inflammation. $\mathrm{J}$ Immunol 182:623-635

92. Staples KJ et al (2012) Phenotypic characterization of lung macrophages in asthmatic patients: overexpression of CCL17. J Allergy Clin Immunol 130:1404-12.e7

93. Panina-Bordignon $\mathrm{P}$ et al (2001) The $\mathrm{C}-\mathrm{C}$ chemokine receptors CCR4 and CCR8 identify airway T cells of allergen-challenged atopic asthmatics. J Clin Investig 107:1357-1364

94. Zhou B et al (2005) Thymic stromal lymphopoietin as a key initiator of allergic airway inflammation in mice. Nat Immunol 6: 1047-1053

95. Besnard A-G et al (2011) IL-33-activated dendritic cells are critical for allergic airway inflammation. Eur J Immunol 41:16751686

96. Claudio E et al (2015) Cutting edge: IL-25 targets dendritic cells to attract IL-9-producing T cells in acute allergic lung inflammation. J Immunol 195:3525-3529

97. Hartl D et al (2006) Chemokines indicate allergic bronchopulmonary aspergillosis in patients with cystic fibrosis. Am J Respir Crit Care Med 173:1370-1376

98. Leung T-F et al (2002) Plasma concentration of thymus and activation-regulated chemokine is elevated in childhood asthma. J Allergy Clin Immunol 110:404-409

99. Bleck B et al (2015) Coexpression of type 2 immune targets in sputum-derived epithelial and dendritic cells from asthmatic subjects. J Allergy Clin Immunol 136:619-627.e5

100. Yoshie O, Matsushima K (2015) CCR4 and its ligands: from bench to bedside. Int Immunol 27:11-20

101. Acosta-Rodriguez EV et al (2007) Surface phenotype and antigenic specificity of human interleukin 17-producing T helper memory cells. Nat Immunol 8:639-646

102. Salimi M et al (2013) A role for IL-25 and IL-33-driven type-2 innate lymphoid cells in atopic dermatitis. J Exp Med 210:29392950

103. Poppensieker K et al (2012) CC chemokine receptor 4 is required for experimental autoimmune encephalomyelitis by regulating GM-CSF and IL-23 production in dendritic cells. Proc Natl Acad Sci U S A 109:3897-3902

104. Bonner K, Pease JE, Corrigan CJ, Clark P, Kay AB (2013) CCL17/thymus and activation-regulated chemokine induces calcitonin gene-related peptide in human airway epithelial cells through CCR4. J Allergy Clin Immunol 132:942-50.e1-3

105. Qi H, Kastenmuller W, Germain RN (2014) Spatiotemporal basis of innate and adaptive immunity in secondary lymphoid tissue. Annu Rev Cell Dev Biol. doi:10.1146/annurev-cellbio-100913013254

106. Foo SY, Phipps S (2010) Regulation of inducible BALT formation and contribution to immunity and pathology. Mucosal Immunol 3: $537-544$
107. GeurtsvanKessel CH et al (2009) Both conventional and interferon killer dendritic cells have antigen-presenting capacity during influenza virus infection. PLoS ONE 4, e7187

108. Halle $\mathrm{S}$ et al (2009) Induced bronchus-associated lymphoid tissue serves as a general priming site for $\mathrm{T}$ cells and is maintained by dendritic cells. J Exp Med 206:2593-2601

109. Hepworth MR et al (2013) Innate lymphoid cells regulate CD4+ T-cell responses to intestinal commensal bacteria. Nature 498: 113-117

110. Hepworth MR et al (2015) Immune tolerance. Group 3 innate lymphoid cells mediate intestinal selection of commensal bacteria-specific $\mathrm{CD}^{+} \mathrm{T}$ cells. Science 348:1031-1035

111. Oliphant CJ et al (2014) MHCII-mediated dialog between group 2 innate lymphoid cells and CD4(+) T cells potentiates type $2 \mathrm{im}-$ munity and promotes parasitic helminth expulsion. Immunity 41: 283-295

112. Phythian-Adams AT et al (2010) CD11c depletion severely disrupts Th2 induction and development in vivo. J Exp Med 207: 2089-2096

113. Smith KA et al (2012) Type 2 innate immunity in helminth infection is induced redundantly and acts autonomously following CD11c(+) cell depletion. Infect Immun 80:3481-3489

114. MacDonald AS, Maizels RM (2008) Alarming dendritic cells for Th2 induction. J Exp Med 205:13-17

115. Willart MAM, Hammad H (2010) Alarming dendritic cells for allergic sensitization. Allergol Int 59:95-103

116. Paul WE (2010) What determines Th2 differentiation, in vitro and in vivo? Immunol Cell Biol 88:236-239

117. Serbina NV, Salazar-Mather TP, Biron CA, Kuziel WA, Pamer EG (2003) TNF/iNOS-producing dendritic cells mediate innate immune defense against bacterial infection. Immunity 19:59-70

118. Schlitzer A, Ginhoux F (2014) Organization of the mouse and human DC network. Curr Opin Immunol 26C:90-99

119. Satpathy AT et al (2012) Zbtb46 expression distinguishes classical dendritic cells and their committed progenitors from other immune lineages. J Exp Med 209:1135-1152

120. Meredith MM et al (2012) Expression of the zinc finger transcription factor zDC (Zbtb46, Btbd4) defines the classical dendritic cell lineage. J Exp Med 209:1153-1165

121. Cisse B et al (2008) Transcription factor E2-2 is an essential and specific regulator of plasmacytoid dendritic cell development. Cell $135: 37-48$

122. Haniffa M et al (2012) Human tissues contain CD141hi crosspresenting dendritic cells with functional homology to mouse CD103+ nonlymphoid dendritic cells. Immunity 37:60-73

123. Jongbloed SL et al (2010) Human CD141+ (BDCA-3) + dendritic cells (DCs) represent a unique myeloid DC subset that crosspresents necrotic cell antigens. J Exp Med 207:1247-1260

124. Schlitzer A et al (2013) IRF4 transcription factor-dependent $\mathrm{CD} 11 \mathrm{~b}+$ dendritic cells in human and mouse control mucosal IL-17 cytokine responses. Immunity 38:970-983

125. Sung S-SJ et al (2006) A major lung CD103 (alphaE)-beta7 integrin-positive epithelial dendritic cell population expressing Langerin and tight junction proteins. J Immunol 176:2161-2172

126. Thornton EE et al (2012) Spatiotemporally separated antigen uptake by alveolar dendritic cells and airway presentation to T cells in the lung. J Exp Med 209:1183-1199

127. Jakubzick C, Helft J, Kaplan TJ, Randolph GJ (2008) Optimization of methods to study pulmonary dendritic cell migration reveals distinct capacities of DC subsets to acquire soluble versus particulate antigen. J Immunol Methods 337:121-131

128. Desch AN et al (2015) Flow cytometric analysis of mononuclear phagocytes in non-diseased human lung and lung-draining lymph nodes. Am J Respir Crit Care Med. doi:10.1164/rccm.201507$1376 \mathrm{OC}$ 
129. Persson EK et al (2013) IRF4 transcription-factor-dependent CD103(+)CD11b(+) dendritic cells drive mucosal T helper 17 cell differentiation. Immunity 38:958-969

130. Gao Y et al (2013) Control of T helper 2 responses by transcription factor IRF4-dependent dendritic cells. Immunity 39:722-732

131. Williams JW et al (2013) Transcription factor IRF4 drives dendritic cells to promote Th2 differentiation. Nat Commun 4:2990

132. Mildner A, Jung S (2014) Development and function of dendritic cell subsets. Immunity 40:642-656

133. Tussiwand $\mathrm{R}$ et al (2015) Klf4 expression in conventional dendritic cells is required for T helper 2 cell responses. Immunity 42:916928

134. Kumamoto $\mathrm{Y}$ et al (2013) $\mathrm{CD}^{2} 01 \mathrm{~b}^{+}$dermal dendritic cells drive $\mathrm{T}$ helper 2 cell-mediated immunity. Immunity 39:733-743

135. Linehan JL et al (2015) Generation of Th17 cells in response to intranasal infection requires TGF- $\beta 1$ from dendritic cells and IL-6 from CD301b + dendritic cells. Proc Natl Acad Sci U S A 112: 12782-12787

136. Everts B et al (2015) Migratory CD103+ dendritic cells suppress helminth-driven type 2 immunity through constitutive expression of IL-12. J Exp Med. doi:10.1084/jem.20150235

137. Swiecki M, Colonna M (2015) The multifaceted biology of plasmacytoid dendritic cells. Nat Rev Immunol. doi:10.1038/ nri3865

138. de Heer HJ et al (2004) Essential role of lung plasmacytoid dendritic cells in preventing asthmatic reactions to harmless inhaled antigen. J Exp Med 200:89-98

139. Lundie RJ et al (2015) A central role for hepatic conventional dendritic cells in supporting Th2 responses during helminth infection. Immunol Cell Biol. doi:10.1038/icb.2015.114

140. Kool M et al (2009) An anti-inflammatory role for plasmacytoid dendritic cells in allergic airway inflammation. J Immunol 183: 1074-1082

141. Becher B et al (2014) High-dimensional analysis of the murine myeloid cell system. Nat Immunol 15:1181-1189

142. Gerner MY, Kastenmuller W, Ifrim I, Kabat J, Germain RN (2012) Histo-cytometry: a method for highly multiplex quantitative tissue imaging analysis applied to dendritic cell subset microanatomy in lymph nodes. Immunity 37:364-376

143. Grainger JR et al (2013) Inflammatory monocytes regulate pathologic responses to commensals during acute gastrointestinal infection. Nat Med 19:713-721

144. Askenase $\mathrm{MH}$ et al (2015) Bone-marrow-resident nk cells prime monocytes for regulatory function during infection. Immunity 42 : $1130-1142$

145. Jakubzick C et al (2013) Minimal differentiation of classical monocytes as they survey steady-state tissues and transport antigen to lymph nodes. Immunity 39:599-610

146. Hondowicz BD et al (2016) Interleukin-2-dependent allergenspecific tissue-resident memory cells drive asthma. Immunity 44:155-166

147. Guo L et al (2015) Innate immunological function of TH2 cells in vivo. Nat Immunol. doi:10.1038/ni.3244

148. Steinman RM, Hawiger D, Nussenzweig MC (2003) Tolerogenic dendritic cells. Annu Rev Immunol 21:685-711

149. Lutz MB (2016) Induction of CD4(+)regulatory and polarized effector/helper T cells by dendritic cells. Immune Netw 16:13-25

150. Maldonado RA, von Andrian UH (2010) How tolerogenic dendritic cells induce regulatory T cells. Adv Immunol 108:111-165

151. Soroosh $\mathrm{P}$ et al (2013) Lung-resident tissue macrophages generate Foxp3+ regulatory T cells and promote airway tolerance. J Exp Med 210:775-788

152. Khare A et al (2013) Cutting edge: inhaled antigen upregulates retinaldehyde dehydrogenase in lung CD103+ but not plasmacytoid dendritic cells to induce Foxp3 de novo in CD4+ T cells and promote airway tolerance. J Immunol 191:25-29
153. Tsoumakidou $\mathrm{M}$ et al (2014) Tolerogenic signaling by pulmonary $\mathrm{CD} 1 \mathrm{c}+$ dendritic cells induces regulatory $\mathrm{T}$ cells in patients with chronic obstructive pulmonary disease by IL-27/IL-10/inducible costimulator ligand. J Allergy Clin Immunol 134:944-954.e8

154. Engler DB et al (2014) Effective treatment of allergic airway inflammation with Helicobacter pylori immunomodulators requires BATF3-dependent dendritic cells and IL-10. Proc Natl Acad Sci U S A 111:11810-11815

155. Quintin J, Cheng S-C, van der Meer JWM, Netea MG (2014) Innate immune memory: towards a better understanding of host defense mechanisms. Curr Opin Immunol 29:1-7

156. Medzhitov R, Schneider DS, Soares MP (2012) Disease tolerance as a defense strategy. Science 335:936-941

157. Mashayekhi M et al (2011) CD8 $\alpha(+)$ dendritic cells are the critical source of interleukin-12 that controls acute infection by Toxoplasma gondii tachyzoites. Immunity 35:249-259

158. Wiesner DL et al (2015) Chitin recognition via chitotriosidase promotes pathologic type-2 helper T cell responses to cryptococcal infection. PLoS Pathog 11, e1004701

159. Hsieh CS et al (1993) Development of TH1 CD4+ T cells through IL-12 produced by Listeria-induced macrophages. Science 260: 547-549

160. Mangan PR et al (2006) Transforming growth factor-beta induces development of the $\mathrm{T}(\mathrm{H}) 17$ lineage. Nature 441:231-234

161. Veldhoen M, Hocking RJ, Atkins CJ, Locksley RM, Stockinger B (2006) TGFbeta in the context of an inflammatory cytokine milieu supports de novo differentiation of IL-17-producing $\mathrm{T}$ cells. Immunity 24:179-189

162. Van Beelen AJ et al (2007) Stimulation of the intracellular bacterial sensor NOD2 programs dendritic cells to promote interleukin17 production in human memory T cells. Immunity 27:660-669

163. Travis MA, Sheppard D (2014) TGF- $\beta$ activation and function in immunity. Annu Rev Immunol 32:51-82

164. Jankovic D, Kullberg M, Caspar P, Sher A (2004) Parasiteinduced Th2 polarization is associated with down-regulated dendritic cell responsiveness to Th1 stimuli and a transient delay in T lymphocyte cycling. J Immunol 173:2419-2427

165. MacDonald AS, Pearce EJ (2002) Cutting edge: polarized Th cell response induction by transferred antigen-pulsed dendritic cells is dependent on IL-4 or IL-12 production by recipient cells. J Immunol 168:3127-3130

166. Perona-Wright G et al (2006) Distinct sources and targets of IL-10 during dendritic cell-driven Th1 and Th2 responses in vivo. Eur J Immunol 36:2367-2375

167. Kool M et al (2011) The ubiquitin-editing protein A20 prevents dendritic cell activation, recognition of apoptotic cells, and systemic autoimmunity. Immunity 35:82-96

168. Cook PC et al (2015) A dominant role for the methyl-CpGbinding protein $\mathrm{Mbd} 2$ in controlling Th2 induction by dendritic cells. Nat Commun 6:6920

169. Heger $\mathrm{K}$ et al (2014) A20-deficient mast cells exacerbate inflammatory responses in vivo. PLoS Biol 12, e1001762

170. Satoh T et al (2010) The Jmjd3-Irf4 axis regulates M2 macrophage polarization and host responses against helminth infection. Nat Immunol. doi:10.1038/ni.1920

171. Hutchins AS et al (2002) Gene silencing quantitatively controls the function of a developmental trans-activator. Mol Cell 10:81-91

172. Chu Y et al (2011) B cells lacking the tumor suppressor TNFAIP3/ A20 display impaired differentiation and hyperactivation and cause inflammation and autoimmunity in aged mice. Blood 117: 2227-2236

173. Licona-Limón P, Kim LK, Palm NW, Flavell RA (2013) TH2, allergy and group 2 innate lymphoid cells. Nat Immunol 14:536-542

174. Chesné J et al (2015) Prime role of IL-17A in neutrophilia and airway smooth muscle contraction in a house dust mite-induced allergic asthma model. J Allergy Clin Immunol 135:1643-1643.e3 